\title{
Transcription Pre-Initiation Complex with TFIIH Reveals Transcription-Ready, Repair-Regulated Helicase Machine from Combined Cryo-EM and Crystallography Datasets
}

\author{
Chunli Yan ${ }^{\mathrm{a}}$, Thomas Dodd ${ }^{\mathrm{a}}$, Yuan He ${ }^{\mathrm{b}}$, Susan E. Tsutakawac ${ }^{\mathrm{c}}$, Ivaylo Ivanovª , John A. Tainer ${ }^{\mathrm{c}, \mathrm{d}}$
}

a. Department of Chemistry and Center for Diagnostics and Therapeutics, Georgia State University, Atlanta, GA 30302, USA, iivanov@gsu.edu

b. Department of Molecular Biosciences and Chemistry of Life Processes Institute, Northwestern University, Evanston, IL 60208, USA, yuanhe@northwestern.edu

c. Molecular Biophysics and Integrated Bioimaging, Lawrence Berkeley National Laboratory, Berkeley, CA 94720, USA, setsutakawa@Ibl.gov

d. Department of Molecular and Cellular Oncology, The University of Texas M. D. Anderson Cancer Center, Houston, TX77030 USA, jatainer@gmail.com

To begin transcription, Pol II depends on key general transcription factors (GTFs: TFIIA, TFIIB, TFIID, TFIIF, TFIIS, TFIIE and TFIIH) that recognize promoter DNA and assemble with the polymerase into a pre-initiation complex (PIC). Furthermore, transcription Factor IIH (TFIIH) plays critical and distinctly different helicase roles in transcription initiation and in Nucleotide Excision Repair. The helicase mechanisms for TFIIH activities in these two pathways are not understood, and published models of TFIIH are missing key parts of TFIIH subunits p62, p52, p44, p34, and XPB. We determined a largely complete TFIIH model based on advanced computational modeling plus integration of published cryoEM maps and crystal structures [1-6]. Strikingly,

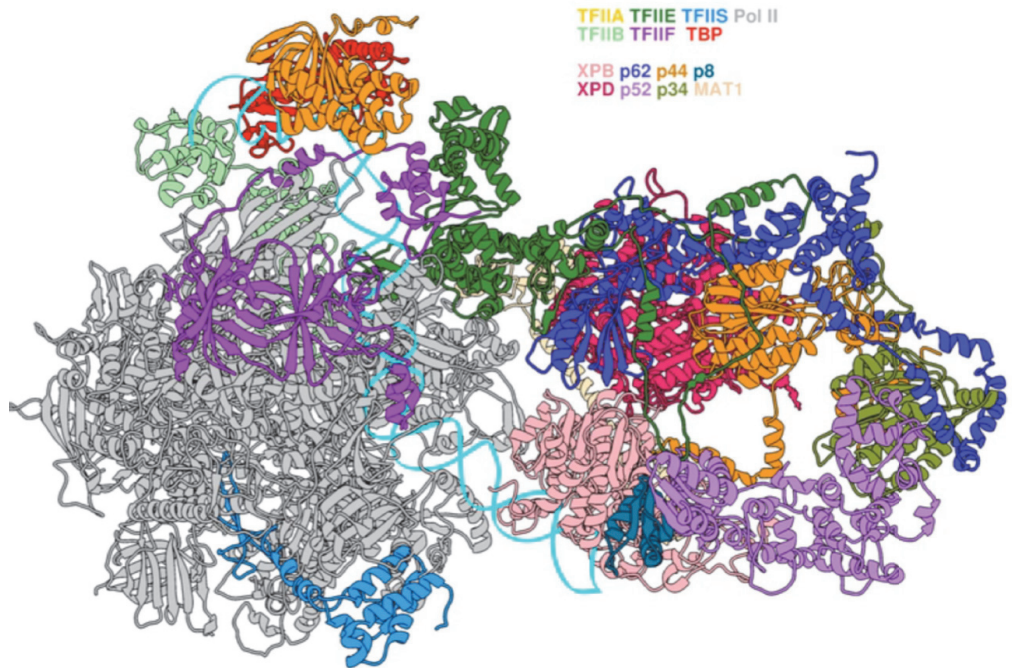

Fig. 1. PIC defined by Cryo-EM, crystallography, and computation. newly modeled regions interlace TFIIH subunits together like "molecular rigging" and are positioned for previously unexpected regulatory functions. The density maps identify key TFIIE/p62 interactions linking core-PIC to TFIIH. Furthermore, p62 caps the ATP binding site in XPD helicase subunit and both XPB and p62 are positioned against the ssDNA binding interface of XPD, indicating that the $X P D$ repair functions are blocked in the apo TFIIH. The mostly complete TFIIH structure enabled molecular dynamics revealing which elements of TFIIH, independent of protein chain, move together as a community or act as "the gears of the TFIIH machine". We find that XPD is a dynamic anchor point and that promoter opening is linked to the global motions and dynamic networks within TFIIH. Notably, 31 of 34 single site patient mutations map to XPD and point to XPD as a central pivot point for TFIIH motions. Overall, the perplexing association of TFIIH with three different diseases is clarified by the finding that disease mutations cluster into distinct classes based on subunit assembly, XPD function, and networked communities.

\section{References}

[1] Fan L, et al. (2006) Mol Cell 22, 27-37.

[2] Fan L, et al. (2008) Cell 133, 789-800.

[3] Fuss JO \&Tainer JA. (2011) DNA Repair (Amst) 10, 697-713.

[4] Mui TP, et al. (2011) J Am Chem Soc. 133,16378-81.

[5] Mason AC, et al. (2014) Proc Natl Acad Sci U S A 111, 7618-23.

[6] Yan' C. et al. (2019) Nature Structure Molecular Biology, in press. 